\title{
Dyslipidemia in chronic renal failure: Cause or effect?
}

\author{
Moushumi Lodh', Sandhya Lal' ${ }^{2}$ Binita Goswami ${ }^{3}$, Partha Karmakar ${ }^{4}$, \\ Ashok Kumar Parida ${ }^{5}$
}

${ }^{1}$ Assistant Professor, Department of Biochemistry, IQ City Medical College and Narayana Multispeciality Hospital, Durgapur, West Bengal, ${ }^{2}$ Registrar, ${ }^{3}$ Associate Professor, Department of Biochemistry, Lady Hardinge Medical College \& Associated Hospitals, New Delhi, ${ }^{4}$ Consultant, Department of Nephrology, Medica Superspeciality Hospital, Kolkata, ${ }^{5}$ Senior Consultant, Department of Cardiology, IQ City Medical College and Narayana Multispeciality Hospital, Durgapur, West Bengal, India

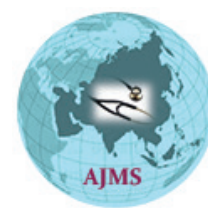

\section{A B S T R A C T}

Background: Various organ systems within the human body are intimately connected to each other. This organ crosstalk mediated by soluble \& cellular mediators help maintain normal homeostasis \& optimum body functioning. But during disease states, this very crosstalk can carry over the influence of the diseased organ to initiate \& perpetuate functional dysfunctions in other organs. Chronic kidney disease may result from different conditions, which may be inherent to the kidney or a consequence of a systemic disease. Aims and Objectives: We conducted this study with the objective to study the metabolic abnormalities in chronic kidney disease patients admitted for dialysis. Such a study has not been documented earlier from this part of India. Materials and Methods: Milky serum of such patients has been recognized as early sign of dyslipidemia. We studied the lipid profile and mineral metabolism in chronic kidney disease and then reviewed all the biochemical pathways involved in etiopathogenesis of renal dyslipidemia. Results: Our study group showed significant hypocalcemia, hypermagnesemia, and hyperphosphatemia, hypoproteinemia, hypoalbuminemia and hyperglobulinemia. The lipid profile was also deranged with statistically significant elevation in total cholesterol and triglyceride levels and significantly lower HDL levels as compared to the controls. Conclusion: Such a study has not been documented earlier from eastern India. The metabolic abnormalities we have observed in chronic kidney disease enhance the risk of atherosclerotic cardiovascular disease in these patients. More prospective studies with larger sample size are required to establish the observations made in this study.

Key words: Renal dyslipidemia, Chronic kidney disease, Azotemia, Hypoalbuminemia, Low HDL, Hypertriglyceridemia

\section{INTRODUCTION}

Chronic Kidney Disease (CKD) has emerged as a health problem with great implications on the economy as well as quality of life of individuals both in India as well as worldwide. ${ }^{1}$ The etiology is multifactorial with the common pathological process involving gradual loss of functional renal capacity leading to widespread biochemical and metabolic perturbations. The management is difficult with the need for renal replacement therapy as hemodialysis or subsequent renal transplantation. According to the Kidney
Disease Outcomes Quality Initiative, chronic kidney disease refers to either kidney damage or a decreased kidney Glomerular Filtration Rate (GFR) of less than $60 \mathrm{ml} / \mathrm{min} / 1.73 \mathrm{~m}^{2}$ for three or more months. ${ }^{2}$

CKD leads to a number of biochemical disturbances which include azotemia, acid base imbalances, dysregulated mineral metabolism and lipid abnormalities. Increase in urea, creatinine and uric acid levels occur due to decreased glomerular filtration. Acidosis is also observed due to the predominant role played by kidneys in

\section{Address for correspondence:}

Dr. Moushumi Lodh, Assistant Professor, Department of Biochemistry, IQ City Medical College and Narayana Multispeciality Hospital,

Durgapur, West Bengal, India. E-mail: drmoushumilodh@gmail.com, Phone: +919800086043 (c) Copyright AJMS 
blood $\mathrm{pH}$ homeostasis. Vitamin $\mathrm{D}$ activation requires 1-alpha hydroxylation that occurs in kidneys. Kidney disease interferes with hydroxylation leading to calcium, magnesium and phosphate metabolism derangements. Lipid alterations are also prevalent in CKD. Amongst the organ crosstalk of various organ systems in the human body, the link between the heart and kidney was recognized and described as early as 60 years ago.

\section{MATERIALS AND METHODS}

The study was conducted in The Mission Hospital, Durgapur, West Bengal, India. The study group comprised of 170 patients with documented CKD who were admitted to the nephrology in- patient department for renal replacement therapy over the period from January 2009-March 2011. The controls were chosen from apparently healthy volunteers among the hospital staff who did not suffer from hypertension, diabetes mellitus, glomerulonephritis, etc. which predispose to impending renal failure.

\section{Inclusion criteria}

Glomerular Filtration Rate (GFR) of less than $60 \mathrm{ml} / \mathrm{min} / 1.73 \mathrm{~m}^{2}$ for three or more months and clinically diagnosed CKD patients posted for hemodialysis or peritoneal dialysis. They were included in the study on the basis of clinical signs and symptoms of kidney disease along with an elevated blood urea and serum creatinine level and a lowered eGFR.

\section{Exclusion criteria}

Chronic kidney disease patients with known cardiac complaints, pregnant ladies, children and CKD patients not requiring dialysis. Institutional ethical clearance was taken and informed consent taken from patients under study.

Blood samples were collected under aseptic conditions after twelve hours of overnight fasting. Samples were then centrifuged, serum separated and biochemical analysis carried out in automated biochemistry analyzers [Siemens dimension expand] using validated commercially available kits. Quality control assessments were done in batch wise assay.

Data was compiled in excel sheet for further statistical analysis. Data was expressed as mean \pm S.E. Normal data was compared between the groups using student's t test and correlation analysis was done to assess the degree of association between the parameters and disease. Statistical analysis was done on Statistical Package for the Social Sciences (SPSS) version 17.0. A p-value $<0.05$ was considered to be significant.

\section{RESULTS}

As is evident from the Table 1, urea, creatinine and uric acid levels were significantly elevated in the CKD patients as compared to the controls which are as expected. The CKD patients demonstrated hypocalcemia, hypermagnesemia, and hyperphosphatemia. This can be explained by the central role of kidneys in mineral metabolism. The patients also exhibited hypoproteinemia, hypoalbuminemia and hyperglobulinemia. Hypoalbuminemia can be explained by the proteinuria that is predominant in CKD patients. Hyperglobulinemia may be due to increased gammaglobulins due to chronic inflammation in such patients. The lipid profile was also deranged in the cases with statistically significant elevation in total cholesterol and triglyceride levels and significantly lower HDL levels as compared to the controls.

Table 2 illustrates the statistically significant correlation analysis of urea with the other parameters in our study. As expected a strong correlation is seen with creatinine levels in the study group. A similar correlation is seen with serum potassium level which is another finding in chronic renal failure (CRF). The negative protein balance

\begin{tabular}{|c|c|c|c|}
\hline \multirow[t]{2}{*}{ Analyte } & \multicolumn{2}{|c|}{ Mean $\pm S . E}$. & \multirow[t]{2}{*}{$P$ value } \\
\hline & Cases & Controls & \\
\hline Urea $(\mathrm{mg} / \mathrm{dl})$ & $104.16 \pm 7.96$ & $25.83 \pm 0.97$ & $<0.001$ \\
\hline Creatinine (mg/dl) & $4.94 \pm 0.51$ & $0.89 \pm 0.03$ & $<0.001$ \\
\hline Uric acid $(\mathrm{mg} / \mathrm{dl})$ & $8.59 \pm 0.41$ & $6.13 \pm 0.18$ & $<0.001$ \\
\hline Sodium $(\mathrm{mmol} / \mathrm{L})$ & $135.47 \pm 0.91$ & $139.83 \pm 1.07$ & 0.006 \\
\hline potassium (mmol/L) & $4.21 \pm 0.14$ & $4.69 \pm 0.10$ & 0.043 \\
\hline chloride (mmol/L) & $98.64 \pm 1.09$ & $104.27 \pm 0.90$ & 0.001 \\
\hline Calcium (mg/dl) & $7.93 \pm 0.30$ & $10.65 \pm 0.16$ & $<0.001$ \\
\hline Phosphorus (mg/dl) & $5.13 \pm 0.40$ & $4.38 \pm 0.21$ & 0.119 \\
\hline Magnesium (mg/dl) & $3.26 \pm 0.34$ & $2.36 \pm 0.05$ & $<0.001$ \\
\hline $\begin{array}{l}\text { Alkaline phosphatase } \\
\text { (mg/dl) }\end{array}$ & $130.45 \pm 19.39$ & $66.83 \pm 3.35$ & 0.015 \\
\hline Total protein $(\mathrm{g} / \mathrm{dl})$ & $6.20 \pm 0.15$ & $7.68 \pm 0.12$ & $<0.001$ \\
\hline Albumin $(\mathrm{g} / \mathrm{dl})$ & $2.54 \pm 0.08$ & $4.58 \pm 0.09$ & $<0.001$ \\
\hline Globulin (g/dl) & $3.64 \pm 0.12$ & $3.10 \pm 0.07$ & 0.001 \\
\hline Cholesterol (mg/dl) & $196.20 \pm 17.90$ & $121.02 \pm 13.19$ & $<0.001$ \\
\hline Triglycerides (mg/dl) & $155.76 \pm 22.5$ & $112.67 \pm 19.1$ & $<0.001$ \\
\hline $\mathrm{HDL}(\mathrm{mg} / \mathrm{dl})$ & $32.7 \pm 8.6$ & $41.2 \pm 7.9$ & $<0.001$ \\
\hline
\end{tabular}

\begin{tabular}{lcc}
$\begin{array}{l}\text { Table 2: Correlation between urea and the } \\
\text { different parameters under study } \\
\text { Parameter }\end{array}$ & $\begin{array}{c}\text { Pearson's correlation } \\
\text { coefficient }\end{array}$ & P value \\
\hline Creatinine & 0.709 & 0.001 \\
Potassium & 0.489 & 0.002 \\
Phosphorus & 0.394 & 0.02 \\
Albumin & -0.336 & 0.02 \\
Cholesterol & 0.678 & 0.01 \\
\hline
\end{tabular}


prevalent in CRF is signified by the negative correlation of urea with serum albumin levels. An interesting finding is the positive correlation between serum cholesterol and urea. This indicates interplay between dyslipidemia and azotemia in CRF.

\section{DISCUSSION}

Chronic Kidney Disease heralds in its wake a plethora of metabolic derangements many of which may be life threatening. We will focus primarily on the lipid abnormalities as many studies have highlighted a causative role of dyslipidemia in CKD. Dyslipidemia is the metabolic consequence of renal failure as well. Hence it will be interesting to evaluate the 'chicken or the egg' dilemma.

We will first consider the lipid abnormalities observed in patients of chronic kidney disorders. The predominant features of 'renal dyslipidemia' are hypertriglyceridemia, hypercholesterolemia and decreased HDL levels. Hypertriglyceridemia may be attributed to increased production coupled with impaired clearance from the body. ${ }^{3}$ Number of biochemical pathways have been implicated in this metabolic derangement. These include decreased lipoprotein lipase (LPL) activity probably due to increased apolipoprotein C-III concentration, presence of yet unidentified inhibitors of lipase activity in the sera of CKD patients and effect of medications on lipoprotein activity. ${ }^{47}$ The activity of hepatic lipase and VLDL receptor and of hepatic LRP is also diminished in CKD. ${ }^{8}$ Schaeffner et $\mathrm{al}^{9}$ also proposed decreased TG degradation result from insufficient mitochondrial beta-oxidation of fatty acids. Proteinuria observed in CKD also leads to upregulation of hepatic DGAT leading to increased triglyceride synthetic capacity. ${ }^{10}$

Some minor contributing pathways have also been mentioned in studies. Secondary hyperparathyroidism is a common entity in CKD. This further impairs catabolism of triglyceride rich lipoproteins. ${ }^{11}$ Moreover increased VLDL synthesis occurs in CKD which can also be resultant of concomitant insulin resistance seen in this condition. ${ }^{12}$

Hypercholesterolemia is a frequent observation in chronic renal failure. Studies have shown that the heavy proteinuria seen in renal failure leads to increased gene expression of HMG-CoA reductase, 7- $\alpha$ hydroxylase and hepatic LDL receptor- important metabolic constituents of in vivo cholesterol homeostasis. ${ }^{13}$ Apolipoprotein B molecules also get modified in CKD due to carbamylation and glycation. This leads to reduced recognition and binding of the apoB containing lipoproteins to LDL receptors and LDL receptor related protein (LRP) in the liver and consequently, reduced plasma clearance occurs. ${ }^{8}$

HDL cholesterol levels are reduced. The pathways implicated include decreased levels of apolipoproteins AI and AII, hepatic lipase deficiency, decreased activity of LCAT, and increased activity of CETP and ACAT being the predominant pathways. ${ }^{14-16}$ Apolipoprotein levels are also altered in CRF with plasma concentrations of apoA-I, apoA-II, and apoE being reduced, whereas that of apoC-III is elevated. Besides affecting plasma lipid levels and their distribution within lipoproteins, CRF affects plasma concentration of various apolipoproteins. ${ }^{5}$ For instance, plasma concentrations of apoA-I, apoA-II, and apoE are reduced, whereas that of apoC-III is elevated and the apoC-II-to-apoC-III concentration ratio is diminished. This further interferes with lipid metabolism. Heavy proteinuria and renal insufficiency in CRF may affect SRB-1 expression and hence, HDL mediated reverse cholesterol transport. ${ }^{10}$ Therapy in chronic renal failure further aggravates the problem. It has been observed that in patients on peritoneal dialysis, peritoneal absorption of glucose tends to accentuate hypertriglyceridemia. ${ }^{17,18}$

We will now appraise about the probable role of dyslipidemia in pathogenesis of CKD. There is growing evidence that circulating lipids are trapped by extracellular matrix molecules in the renal glomerulus. ${ }^{19}$ These undergo oxidation to generate free radicals. These start an oxidation cascade that interferes with the functioning of endothelium derived vasodilators such as NO, prostacyclins, and vasodialators such as endothelin-1, angiotensin II, plasminogen activator inhibitor- 1 and others. This disturbs the delicate balance that exists between vasodilator and vasoconstrictive forces leading to endothelial dysfunction. Macrophages in the renal interstitium get transformed into foam cells that release a repertoire of cytokines which have profound implications on vascular homeostasis. These pathological changes mimic atherosclerosis which initiates and aggravates renal dysfunction. ${ }^{20,21}$ Also a secondary form of dyslipidemia occurs in patients with chronic disease that mimics the atherogenic dyslipidemia of insulin-resistant patients. This suggests possible role of insulin resistance syndrome in association between dyslipidemia and loss of renal function. ${ }^{22}$

Furthermore, dyslipidemia has been associated with glomerular capillary damage and mesangial and podocyte injury. LDL receptors are expressed on mesangial cells which engulf LDL and oxidized LDL. This activates mesangial cell proliferation and expression of chemokines and proinflammatory cytokines that initiate growth. Oxidised LDL also induces podocyte apoptosis, nephrin loss and increased permeability to albumin. Oxidized 
LDL also increases monocyte adhesion, infiltration and migration. ${ }^{23}$ There is increasing evidence that reabsorption of lipids in the renal matrix may precipitate tubulointerstitial inflammation, glomerulosclerosis and tissue injury. Low HDL seen in dyslipidemia further interferes with reverse cholesterol transport. Infact, low HDL has been associated with progressive renal disease. ${ }^{24}$ Many large scale prospective studies such as ARIC study, Framingham Offspring study, Physicians' Health Study have established the association between dyslipidemia and progressive renal diseases. ${ }^{25}$ In the Modification of Diet in Renal Disease study, which included patients with moderate-to-severe renal disease of various etiologies, low HDL independently predicted a faster decline in GFR. ${ }^{26}$ Treatment with statins has been shown to decrease the progression of renal failure in the SHARP trial, thereby proving the role of lipids in renal failure. ${ }^{27}$

\section{CONCLUSION}

From the study it can be concluded that CKD patients show abnormal lipid profile in the cases with statistically significant elevation in total cholesterol and triglyceride levels and significantly lower HDL levels as compared to the controls.

The CKD patients also demonstrated hypocalcemia, hypermagnesemia, and hyperphosphatemia as kidneys play central role in mineral metabolism. The patients also exhibited hypoproteinemia, hypoalbuminemia and hyperglobinemia. Hypoalbuminemia can be explained by the heavy proteinuria that is predominant in CKD patients. Hyperglobinemia observed can be explained by chronic inflammation leading to increased gammaglobulins in such patients. Together, these abnormalities enhance the risk of atherosclerotic cardiovascular disease in these patients. A prospective study with larger sample size is planned to establish the observations made in this study.

\section{REFERENCES}

1. Raju SSK, Lalitha DL and Kiranmayi P. A study of lipid profile and lipid peroxidation in chronic kidney disease with special reference to hemodialysis. Clinical Research \& Bioethics 2013; 4(1): $1-5$.

2. Levey AS, Eckardt KU, Tsukamato Y, Levin A, Coresh J, Rossert J, et al. Definition and classification of chronic kidney disease: a positive statement from kidney disease: positive outcomes (KDIGO). Kidney Int 2005; 67: 2089-2100.

3. Merzah KS and Hasson SF. The Biochemical changes in patients with chronic renal failure. International Journal of Pharma Medicine and Biological Sciences 2015; 4(1):75-79.

4. Vaziri ND and Liang K. Down-regulation of tissue lipoprotein lipase expression in experimental chronic renal failure. Kidney International 1996; 50 (6): 1928-1935.
5. Moberly JB, Attman PO, Sameulssen O, Johannsen AC, Kight- Gibson C and Alaupovic P. Apolipoprotein CIII, hypertriglyceridemia and triglyceride-rich lipoproteins in uremia. Miner Electrolyte Metab 1999; 25:258-262.

6. Cheung AK, Parker CJ, Ren K and Iverius PH. Increased lipase inhibition in uremia: identification of pre beta HDL as a major inhibitor in normal and uremic plasma. Kidney International 1996; 49: 1360-1371.

7. Rao AM, Bitla AR, Reddy EP, Sivakumar V and Rao PVLNS. Lipid abnormalities, lipoprotein (a), apoprotein pattern in nondialyzed patients with chronic kidney disease. IJCB 2010; 25(1): 47-50.

8. Vaziri ND. Dyslipidemia of chronic renal failure: the nature, mechanisms, and potential consequences. Am J Physiol Renal Physiol 2006; 290: F262-F272.

9. Schaefer F, Chen $Y$, Tsao T, Nouri P and Rabkin R. Impaired JAK-STAT signal transduction contributes to growth hormone resistance in chronic uremia. The Journal of Clinical Investigation 2001; 108: 467-475.

10. Seiber $\mathrm{J}$ and Jelhe AW. Free fatty acids and their metabolism affect function and survival of podocytes. Front Endocrinol 2014; 186(5): 1-7.

11. Tsimihodimos V, Mitrogianni Z and Elisaf M. Dyslipidemia associated with kidney disease. Open Cardiovasc Med J 2011; 5: 41-48.

12. Mesquita J, Varela A and Medina JL. Dyslipidemia in renal disease: Causes, consequences and treatment. Endocrinol Nutr 2010; 57(9): 440-448.

13. Mohanraj $P$, Anbazhagan $G$ and Kalaivalli $S$. Evaluation of Lipid Profile in Non-Diabetic Chronic Kidney Disease Stage 3 and 4. Journal of Evidence Based Medicine and Healthcare 2014; 1(6): 338-346.

14. Fielding $\mathrm{CJ}$ and Fielding PE.Cellular cholesterol efflux. Biochim Biophys Acta 2001; 1533: 175-189.

15. De Sain-van der Velden MG, Rabelink TJ, Reijngoud DJ, Gadelha MM, Voorbij HA, Stellaard F, et al. Plasma alpha 2 macroglobulin is increased in nephritic patient as a result of increased synthesis alone. Kidney International 1998; 54: 530-535.

16. Oram JF. Tangier disease and ABCA1. Biochim Biophys Acta 2000; 1529: 321-330.

17. Kanda E, Ai M, Okazaki M, Maeda Y, Sasaki S and Yoshida M. The association of very low density lipoprotein with ankle brachial index in peritoneal dialysis patients with controlled serum low density lipoprotein cholesterol level. BMC Nephrology 2013; 14:212.

18. Sreenivasulu $U$, Bhagyamma $S N$ and Anuradha R. Study of Lipid Profile in Chronic Renal Failure Patients Undergoing Haemodialysis: A Hospital Based Study. Journal of Evidence based Medicine and Healthcare 2015; 45(2): 8131-8135.

19. Trevisan R, Dodesini AR and Lepore G. Lipids and renal disease. J Am Soc Nephrol 2006; 17: S145-S147.

20. Chait $A$ and Heinecke JW. Lipoprotein modification: cellular mechanisms. Current Opin Lipidol 1994; 5: 363-370.

21. Wheeler DC and Chana RS. Interaction between lipoproteins, glomerular cells and matrix. Miner Electrolyte Metab 1993; 19:149-164

22. Kaur J. A comprehensive review on metabolic syndrome. Cardiol Res Pract 2014; 2014: 943162.

23. Deregibus MC, Fonsato V, Doublier S, Spatola T, Procida S, Di Carlo FD, et al. Statins prevent oxidized LDL-induced injury of glomerular podocytes by activating the Phosphatidylinositol 3-Kinase/AKT-signaling pathway. J Am Soc Nephrol 2005; 6:1936-1947.

24. Cases A and Coll E. Dyslipidemia and progression of renal 
disease in chronic renal failure. Kidney International 2005; 68: S87-S93.

25. Anderson TJ, Gregoire J, Hegele RA, Couture P, Mancini J, McPherson R, et al. Update of the Canadian Cardiovascular Society Guidelines for the Diagnosis and Treatment of Dyslipidemia for the Prevention of Cardiovascular Disease in the Adult. Can J Cardiol 2013; 29:151-167.
26. Nashar K and Egan BM. Relationship between chronic kidney disease and metabolic syndrome: current perspectives. Diabetes Metab Syndr Obes 2014; 7:421-435.

27. Naderi $S$ and Foody JM. Ezetimibe and simvastatin for the prevention of cardiovascular events in predialysis chronic kidney disease patients: a review. Int J Nephrol Renovasc 2012; 5: 165-169.

\section{Authors Contribution:}

ML, AKP, BG, and PK - Concept, design, manuscript preparation, editing and review, ML, SL - Data analysis.

Source of Support: Nil, Conflict of Interest: None declared. 